\title{
The genome sequence of the common toad, Bufo bufo
}

\section{(Linnaeus, 1758) [version 1; peer review: 2 approved]}

\section{Jeffrey W. Streicher (D1), Wellcome Sanger Institute Tree of Life programme, Wellcome Sanger Institute Scientific Operations: DNA Pipelines collective, Tree of Life Core Informatics collective, Darwin Tree of Life Consortium}

${ }^{1}$ Department of Life Sciences, Natural History Museum, London, UK

V1 First published: 20 Oct 2021, 6:281

https://doi.org/10.12688/wellcomeopenres.17298.1

Latest published: 20 Oct 2021, 6:281

https://doi.org/10.12688/wellcomeopenres.17298.1

\section{Abstract}

We present a genome assembly from an individual male Bufo bufo (the common toad; Chordata; Amphibia; Anura; Bufonidae). The genome sequence is 5.04 gigabases in span. The majority of the assembly (99.1\%) is scaffolded into 11 chromosomal pseudomolecules. Gene annotation of this assembly by the NCBI Eukaryotic Genome Annotation Pipeline has identified 21,517 protein coding genes.

Keywords

Bufo bufo, common toad, genome sequence, chromosomal

This article is included in the Tree of Life gateway.

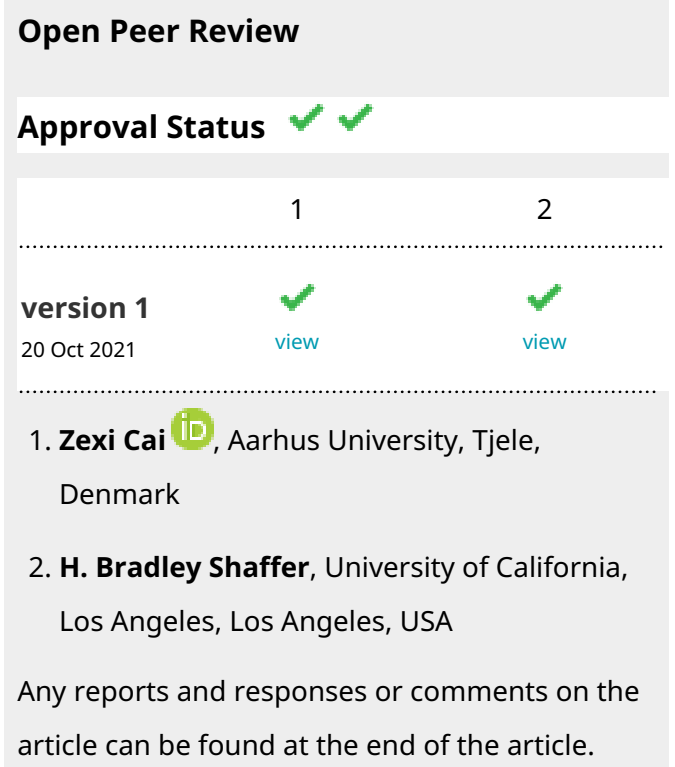

Corresponding author: Darwin Tree of Life Consortium (mark.blaxter@sanger.ac.uk)

Author roles: Streicher JW: Investigation, Resources, Writing - Original Draft Preparation, Writing - Review \& Editing;

Competing interests: No competing interests were disclosed.

Grant information: This work was supported by the Wellcome Trust through core funding to the Wellcome Sanger Institute (206194) and the Darwin Tree of Life Discretionary Award (218328).

The funders had no role in study design, data collection and analysis, decision to publish, or preparation of the manuscript.

Copyright: ( 2021 Streicher JW et al. This is an open access article distributed under the terms of the Creative Commons Attribution License, which permits unrestricted use, distribution, and reproduction in any medium, provided the original work is properly cited.

How to cite this article: Streicher JW, Wellcome Sanger Institute Tree of Life programme, Wellcome Sanger Institute Scientific Operations: DNA Pipelines collective et al. The genome sequence of the common toad, Bufo bufo (Linnaeus, 1758) [version 1; peer review: 2 approved] Wellcome Open Research 2021, 6:281 https://doi.org/10.12688/wellcomeopenres.17298.1

First published: 20 Oct 2021, 6:281 https://doi.org/10.12688/wellcomeopenres.17298.1 


\section{Species taxonomy}

Eukaryota; Metazoa; Chordata; Craniata; Vertebrata; Euteleostomi; Amphibia; Batrachia; Anura; Neobatrachia; Hyloidea; Bufonidae; Bufo; Bufo bufo Linnaeus 1758 (NCBI:txid8384).

\section{Introduction}

The common toad, Bufo bufo (Anura: Bufonidae) is widely distributed throughout Europe. It has a biphasic life cycle that includes aquatic, benthic larvae and terrestrial adults. Bufonids like $B$. bufo are notable amongst anurans in that they (1) lack maxillary teeth, (2) have Bidder's organs, and (3) have paired paratoid glands that contain alkaloid toxins. Bufo bufo has been used extensively in comparative vertebrate research including as a model system in sensory biology (Ewert, 1974).

Based on populations from mainland Europe, the nuclear genome size of $B$. bufo was previously estimated to be between 5.82 and 7.75 picograms (= 5.69 and 7.58 gigabases; (Gregory, 2021)). This is slightly larger than our 5.04 gigabase assembly. The eleven pseudomolecules in our assembly match the expected number of chromosomes in B. bufo $(2 \mathrm{~N}=22$; six macro- and five micro-chromosomes; (Birstein \& Mazin, 1982; Makino \& Others, 1951).

This is the third nuclear genome sequence to be reported from a bufonid anuran (Edwards et al., 2018; Lu et al., 2021). The B. bufo reference genome reported here has been used to study pseudogenization of the tooth enamel gene amelogenin in bufonids (Shaheen et al., 2021). The genome of a common toad from the UK is particularly timely as a tool for understanding the dynamics of population declines observed over the last two decades (Carrier \& Beebee, 2003; Petrovan \& Schmidt, 2016).

\section{Genome sequence report}

The genome was sequenced from one male B. bufo collected from the Natural History Museum (NHM) Wildlife Garden, London, UK (Figure 1A, B). A total of 64-fold coverage in Pacific Biosciences single-molecule long reads (N50 $28 \mathrm{~kb}$ ) and 56-fold coverage in 10X Genomics read clouds (from molecules with an estimated N50 of $29 \mathrm{~kb}$ ) were generated. Primary assembly contigs were scaffolded with chromosome conformation Hi-C data. Manual assembly curation corrected 3498 missing/misjoins and removed 513 haplotypic duplications, reducing the assembly length by $2.4 \%$ and the scaffold number by $49.5 \%$, and increasing the scaffold N50 by $38.9 \%$.

The final assembly has a total length of $5.04 \mathrm{~Gb}$ in 1307 sequence scaffolds with a scaffold N50 of $636 \mathrm{Mb}$ (Table 1). The majority, $99.1 \%$, of the assembly sequence was assigned to 11 chromosomal-level scaffolds (numbered by sequence length) (Figure 2-Figure 5; Table 2). The assembly has a BUSCO (Simão et al., 2015) v5.1.2 completeness of $90.1 \%$ using the tetrapoda_odb10 reference set. However, a BUSCO (v4.0.2) score of $95.3 \%$ using the same reference set was obtained for the annotated gene set of the aBufBuf1.1 assembly (see section Genome annotation), indicating that the assembly has a high level of completeness and that some genes were missed during BUSCO analysis of the whole genome assembly. While not fully phased, the assembly deposited is of one haplotype. Contigs corresponding to the second haplotype have also been deposited.

\section{Genome annotation}

The B. bufo assembly was annotated by the NCBI Eukaryotic Genome Annotation Pipeline, an automated pipeline that
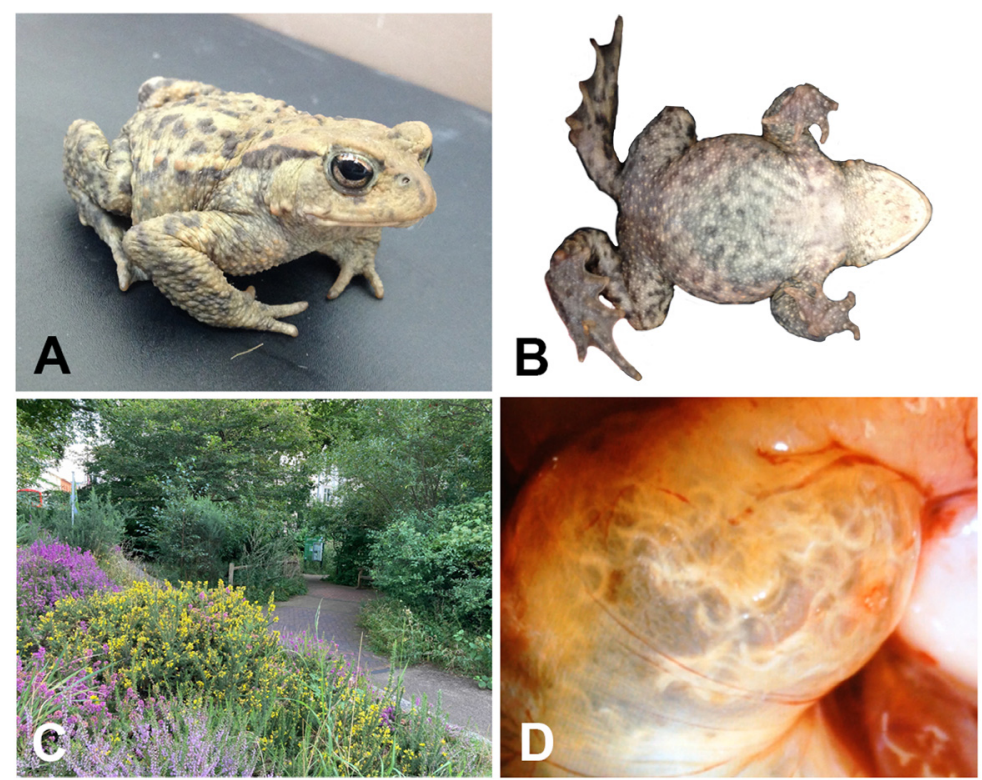

Figure 1. (A) Male voucher specimen of Bufo bufo (NHMUK 2013.484; Field ID, JWS 758; Snout-Vent Length $55.5 \mathrm{~mm}$ ) from which the genome was sequenced. (B) Ventral surface of NHMUK 2013.484. (C) The individual was collected from the Natural History Museum Wildlife Garden, London, England. (D) Large numbers of an unidentified nematode parasite were present in the stomach of NHMUK 2013.484. 
Table 1. Genome data for Bufo bufo, aBufBuf1.1.

\begin{tabular}{|c|c|}
\hline \multicolumn{2}{|l|}{ Project accession data } \\
\hline Assembly identifier & aBufBuf1.1 \\
\hline Species & Bufo bufo \\
\hline Specimen & aBufBuf1 \\
\hline NCBI taxonomy ID & NCBI:txid8384 \\
\hline BioProject & PRJEB42238 \\
\hline BioSample ID & SAMEA7521636 \\
\hline Isolate information & Male, heart tissue; NHMUK 2013.484 \\
\hline \multicolumn{2}{|l|}{ Raw data accessions } \\
\hline PacificBiosciences SEQUEL II & ERR7012639, ERR7015063-ERR7015065 \\
\hline 10X Genomics Illumina & ERR6002753-ERR6002766, ERR6003048, ERR6003049 \\
\hline Hi-C Illumina & ERR6002767-ERR6002770 \\
\hline BioNano & ERZ3003198 \\
\hline \multicolumn{2}{|l|}{ Genome assembly } \\
\hline Assembly accession & GCA_905171765.1 \\
\hline Accession of alternate haplotype & GCA_905171715.1 \\
\hline Span (Mb) & 5,045 \\
\hline Number of contigs & 5,502 \\
\hline Contig N50 length (Mb) & 3.96 \\
\hline Number of scaffolds & 1,307 \\
\hline Scaffold N50 length (Mb) & 636 \\
\hline Longest scaffold (Mb) & 843 \\
\hline BUSCO genome score* & C:90.1\%[S:88.5\%,D:1.6\%],F:3.2\%,M:6.7\%,n:5310 \\
\hline \multicolumn{2}{|l|}{ Genome annotation } \\
\hline Number of genes & 30,286 \\
\hline $\begin{array}{l}\text { Number of protein-coding } \\
\text { genes }\end{array}$ & 21,517 \\
\hline Average length of gene (bp) & 57,667 \\
\hline $\begin{array}{l}\text { Average number of exons per } \\
\text { gene }\end{array}$ & 12 \\
\hline Average exon size (bp) & 241 \\
\hline Average intron size (bp) & 8,995 \\
\hline BUSCO annotation score ${ }^{* *}$ & C:95.3\%[S:93.2\%,D:2.1\%],F:0.7\%,M:4.0\%,n:5310 \\
\hline
\end{tabular}

$\mathrm{C}=$ complete $[\mathrm{S}=$ single copy, $\mathrm{D}=$ duplicated], $\mathrm{F}=$ fragmented, $\mathrm{M}=$ =missing, $\mathrm{n}=$ number of orthologues in comparison.

*BUSCO scores based on the terapoda_odb10 BUSCO set using v5.1.2, run on the aBufBuf1.1 genome assembly using BlobToolKit. A full set of BUSCO scores is available at https://blobtoolkit.genomehubs. org/view/aBufBuf1.1/dataset/CAJIMN01/busco.

**BUSCO scores based on the terapoda_odb10 BUSCO set using v4.0.2, run on the NCBI RefSeq annotation of the aBufBuf1.1 genome assembly (NCBI Bufo bufo Annotation Release 100). 
Scaffold statistics

BUSCO tetrapoda_odb10(5310)

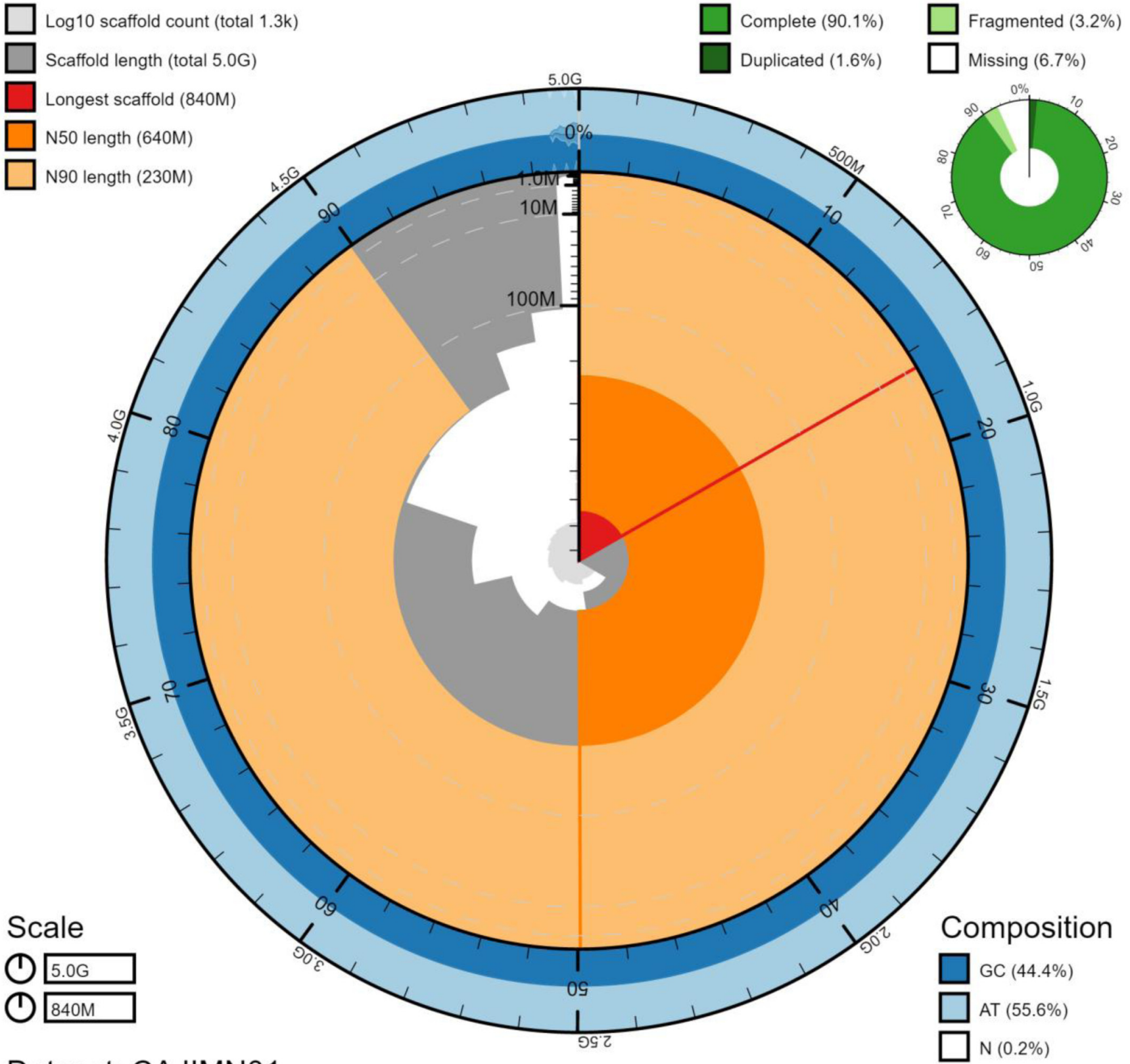

\section{Dataset: CAJIMN01}

Figure 2. Genome assembly of Bufo bufo, aBufBuf1.1: metrics. The BlobToolKit Snailplot shows N50 metrics and BUSCO gene completeness. The main plot is divided into 1,000 size-ordered bins around the circumference with each bin representing $0.1 \%$ of the $5,044,762,059 \mathrm{bp}$ assembly. The distribution of chromosome lengths is shown in dark grey with the plot radius scaled to the longest chromosome present in the assembly $(843,366,180 \mathrm{bp}$, shown in red). Orange and pale-orange arcs show the N50 and N90 chromosome lengths $(635,713,434$ and $230,778,867$ bp), respectively. The pale grey spiral shows the cumulative chromosome count on a log scale with white scale lines showing successive orders of magnitude. The blue and pale-blue area around the outside of the plot shows the distribution of GC, AT and N percentages in the same bins as the inner plot. A summary of complete, fragmented, duplicated and missing BUSCO genes in the tetrapoda_odb10 set is shown in the top right. An interactive version of this figure is available at https://blobtoolkit.genomehubs. org/view/Bufo\%20bufo/dataset/CAJIMN01/snail. 


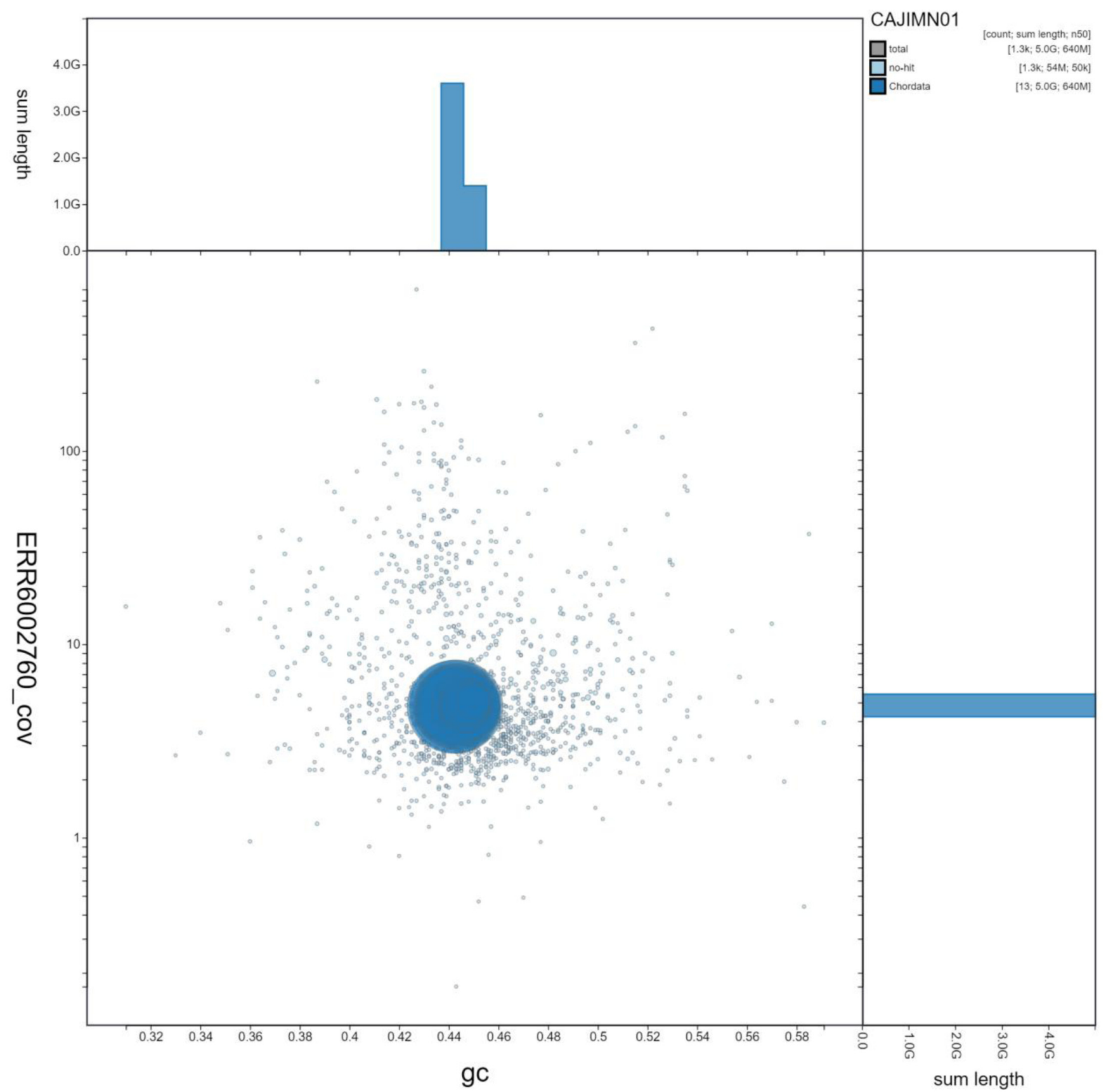

Figure 3. Genome assembly of Bufo bufo, aBufBuf1.1: GC-coverage. BlobToolKit GC-coverage plot. Scaffolds are coloured by phylum. Circles are sized in proportion to scaffold length. Histograms show the distribution of scaffold length sum along each axis. An interactive version of this figure is available at https://blobtoolkit.genomehubs.org/view/Bufo\%20bufo/dataset/CAJIMN01/blob.

annotates genes, transcripts and proteins on draft and finished genome assemblies. The annotation (NCBI Bufo bufo Annotation Release 100; Table 1) was generated from transcripts and proteins retrieved from NCBI Entrez by alignment to the genome assembly, as described (Pruitt et al., 2014).

\section{Methods}

Sample acquisition

A single male $B$. bufo was collected from a stable, isolated population in the NHM Wildlife Garden, London, UK (latitude 51.49586, longitude -0.178622 , elevation $17 \mathrm{~m}$ ) by Jeffrey 


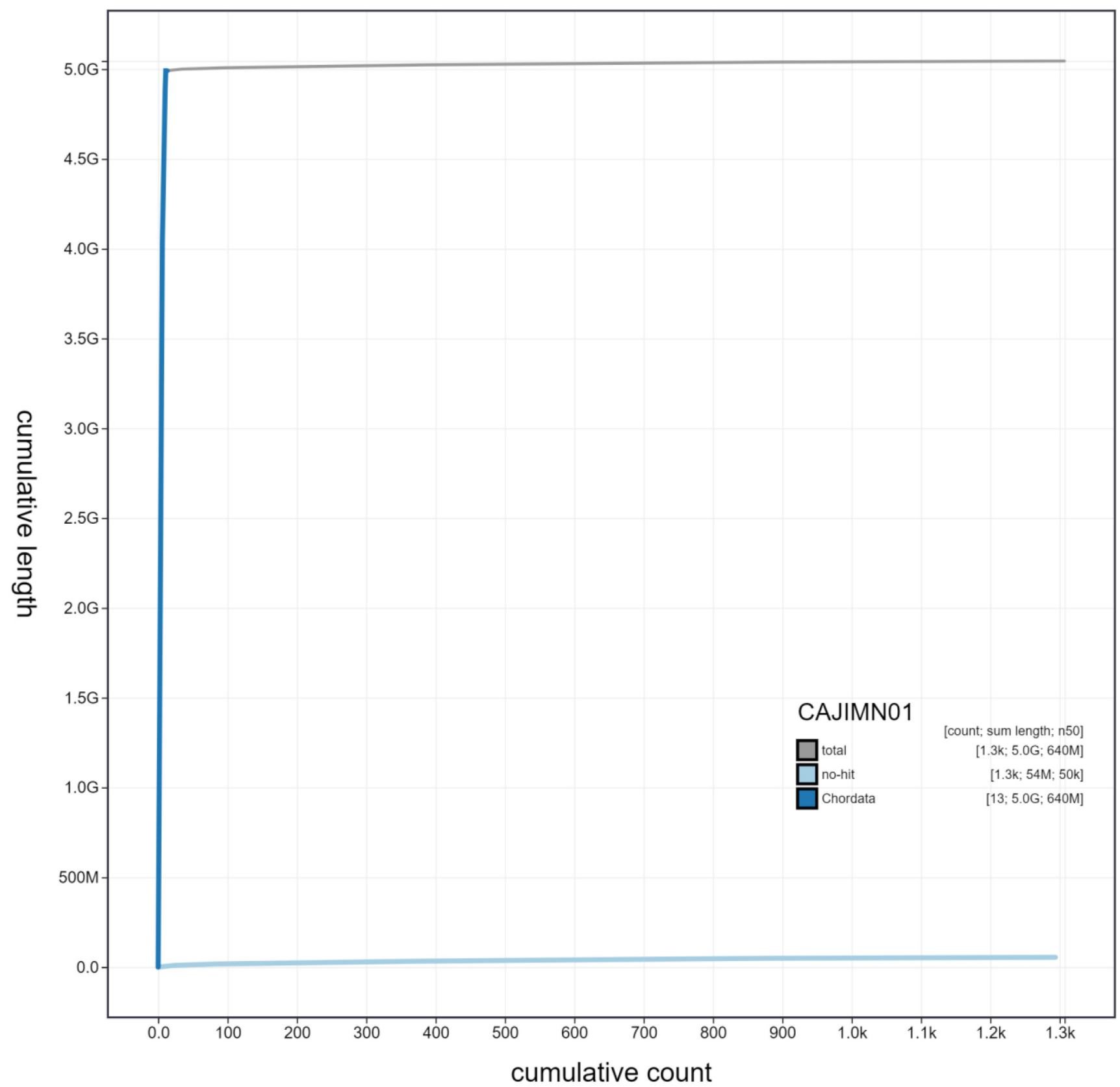

Figure 4. Genome assembly of Bufo bufo, aBufBuf1.1: cumulative sequence. BlobToolKit cumulative sequence plot. The grey line shows cumulative length for all scaffolds. Coloured lines show cumulative lengths of scaffolds assigned to each phylum using the buscogenes taxrule. An interactive version of this figure is available at https://blobtoolkit.genomehubs.org/view/Bufo\%20bufo/dataset/ CAJIMN01/cumulative.

W. Streicher on 1 July 2015 (Figure 1C). The specimen of B. bufo (NHMUK 2013.484, Field ID: JWS 758) was $55.5 \mathrm{~mm}$ snout-vent length (determined using Miyamoto digital callipers to the nearest $0.1 \mathrm{~mm}$ ) and contained many nematode parasites in its stomach (Figure 1D). The specimen was collected with permission from the NHM Wildlife Garden management team and is part of a long-term monitoring project run by the
Department of Life Sciences and the Angela Marmont Centre for UK Biodiversity. It was humanely euthanised using a saturated solution of tricaine mesylate (MS-222). Multiple tissues including heart, thigh muscle, liver, eyes, kidney, testes, Bidder's organ, and intestines were sampled into an ammonium sulfate-based RNA + DNA preservation buffer. After $\sim 24$ hours of storage at $4^{\circ} \mathrm{C}$, the tissues were transferred 


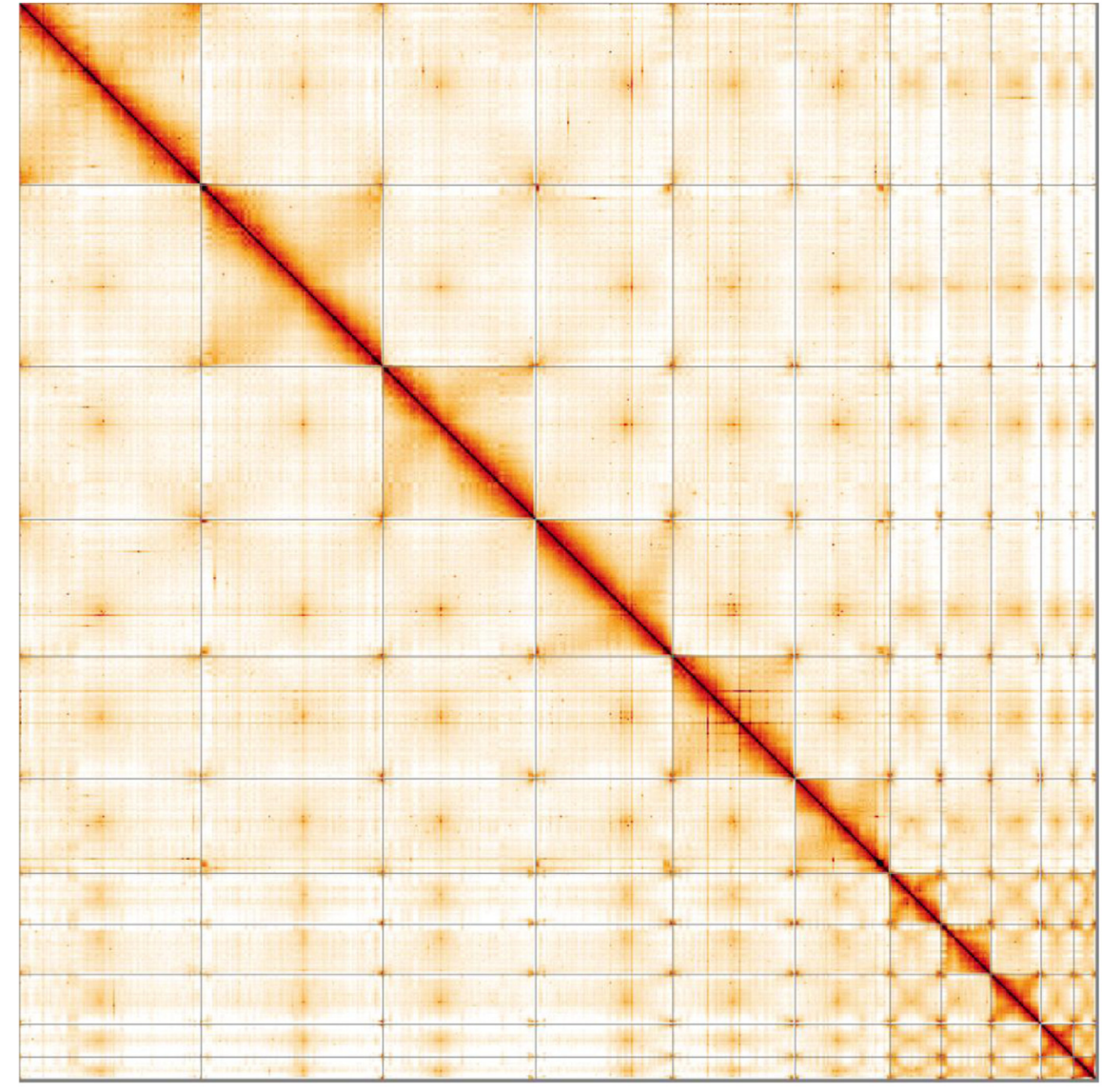

Figure 5. Genome assembly of Bufo bufo, aBufBuf1.1: Hi-C contact map. Hi-C contact map of the aBufBuf1.1 assembly, visualised in HiGlass. Chromosomes are presented in size order from left to right and top to bottom.

\begin{tabular}{|c|c|c|c|}
\hline INSDC accession & Chromosome & Size (Mb) & GC\% \\
\hline LR991667.1 & 1 & 843.37 & 44.5 \\
\hline LR991668.1 & 2 & 842.56 & 44.3 \\
\hline LR991669.1 & 3 & 707.96 & 44.4 \\
\hline LR991670.1 & 4 & 635.71 & 44.4 \\
\hline LR991671.1 & 5 & 567.30 & 44.4 \\
\hline LR991672.1 & 6 & 439.63 & 44.8 \\
\hline LR991673.1 & 7 & 236.60 & 44.7 \\
\hline LR991674.1 & 8 & 231.67 & 44.8 \\
\hline LR991675.1 & 9 & 230.78 & 44.8 \\
\hline LR991676.1 & 10 & 151.57 & 44.8 \\
\hline LR991677.1 & 11 & 103.21 & 45 \\
\hline LR991678.1 & MT & 0.02 & 42.7 \\
\hline- & Unlocalised & 54.40 & 45.1 \\
\hline
\end{tabular}


to $-80^{\circ} \mathrm{C}$ until they were sent for genome sequencing. Sample tissue has been accessioned by the NHM Molecular Collections Facility (NHMUK 2013.484).

\section{DNA extraction and sequencing}

DNA was extracted from heart tissue using the Bionano Prep Animal Tissue DNA Isolation kit according to the manufacturer's instructions. Pacific Biosciences CLR long read and 10X Genomics read cloud sequencing libraries were constructed according to the manufacturers' instructions. Hi-C data were generated from heart tissue using the Arima v2 Hi-C kit. Extraction and sequencing was performed by the Scientific Operations DNA Pipelines at the Wellcome Sanger Institute on Pacific Biosciences SEQUEL I and Illumina HiSeq $\mathrm{X}$ instruments. DNA was labeled for Bionano Genomics optical mapping following the Bionano Prep Direct Label and Stain (DLS) Protocol and run on one Saphyr instrument chip flowcell.

\section{Genome assembly}

Assembly was carried out following the Vertebrate Genome Project pipeline v1.6 ((Rhie et al., 2021)) with Falcon-unzip (Chin et al., 2016), haplotypic duplication was identified and removed with purge_dups (Guan et al., 2020) and a first round of scaffolding carried out with $10 \mathrm{X}$ Genomics read clouds using scaff10x. Hybrid scaffolding was performed using the BioNano DLE-1 data and BioNano Solve. Scaffolding with Hi-C data ((Rao et al., 2014)) was carried out with HiLine, then 3D-DNA (Dudchenko et al., 2017). The Hi-C scaffolded assembly was polished with arrow using the PacBio data, then polished with the 10X Genomics Illumina data by aligning to the assembly with longranger align, calling variants with freebayes (Garrison \& Marth, 2012) and applying homozygous non-reference edits using bcftools consensus. Two rounds of the Illumina polishing were applied. The mitochondrial genome was assembled at The Rockefeller University using the mitoVGP pipeline (Formenti et al., 2021). The assembly was checked for contamination and corrected using the gEVAL system (Chow et al., 2016; (Howe et al., 2021)). Manual curation was performed using evidence from Bionano (using the Bionano Access viewer), using HiGlass (Kerpedjiev et al., 2018) and Pretext, as described previously (Howe et al., 2021). Figure 2-Figure 4 and BUSCO values were generated using BlobToolKit (Challis et al., 2020). Table 3 contains a list of software tools and versions, where applicable.

\section{Ethical/compliance issues}

The materials that have contributed to this genome note were supplied by a Tree of Life collaborator. The Wellcome Sanger Institute employs a process whereby due diligence is carried out proportionate to the nature of the materials themselves, and the circumstances under which they have been/are to be collected and provided for use. The purpose of this is to address and mitigate any potential legal and/or ethical implications of receipt and use of the materials as part of the research project, and to ensure that in doing so we align with best practice wherever possible.

Table 3. Software tools used.

\begin{tabular}{|l|l|l|}
\hline Software tool & Version & Source \\
\hline Falcon-unzip & falcon-kit 1.4.2 & (Chin et al., 2016) \\
\hline purge_dups & 1.0 .0 & (Guan et al., 2020) \\
\hline scaff10x & 4.2 & https://github.com/wtsi-hpag/Scaff10X \\
\hline Bionano Solve & 3.3_10252018 & https://bionanogenomics.com/downloads/bionano-solve/ \\
\hline 3D-DNA & 180922 & (Dudchenko et al., 2017) \\
\hline Arrow & $\begin{array}{l}\text { gcpp 1.9.0-SL-release- } \\
\text { 8.0.0+1-37-gd7b188d }\end{array}$ & https://github.com/PacificBiosciences/GenomicConsensus \\
\hline Longranger & 2.2 .2 & $\begin{array}{l}\text { https://support.10xgenomics.com/genome-exome/ } \\
\text { software/pipelines/latest/advanced/other-pipelines }\end{array}$ \\
\hline freebayes & v1.3.1-17-gaa2ace8 & (Garrison \& Marth, 2012) \\
\hline bcftools- & 1.10 .2 & http://samtools.github.io/bcftools/bcftools.html \\
consensus & N/A & (Chow et al., 2016) \\
\hline gEVAL & 1.11 .6 & (Kerpedjiev et al., 2018) \\
\hline HiGlass & 0.1 & https://github.com/wtsi-hpag/PretextView \\
\hline PretextView & 2.6 .1 & (Challis et al., 2020) \\
\hline BlobToolKit & &
\end{tabular}


The overarching areas of consideration are:

- Ethical review of provenance and sourcing of the material;

- Legality of collection, transfer and use (national and international).

Each transfer of samples is undertaken according to a Research Collaboration Agreement or Material Transfer Agreement entered into by the Tree of Life collaborator, Genome Research Limited (operating as the Wellcome Sanger Institute) and in some circumstances other Tree of Life collaborators.

\section{Data availability}

European Nucleotide Archive: Bufo bufo (common toad). Accession number PRJEB42238; https://identifiers.org/ena.embl/ PRJEB42238.

The genome sequence is released openly for reuse. The $B$. bufo genome sequencing initiative is part of the Darwin Tree of Life (DToL) project and the Vertebrate Genomes Project. All raw sequence data and the assembly have been deposited in INSDC databases. Raw data and assembly accession identifiers are reported in Table 1 .

\section{Author information}

Members of the Wellcome Sanger Institute Tree of Life programme collective are listed here: https://doi.org/10.5281/ zenodo. 5377053 .

Members of Wellcome Sanger Institute Scientific Operations: DNA Pipelines collective are listed here: https://doi.org/10.5281/ zenodo.4790456.

Members of the Tree of Life Core Informatics collective are listed here: https://doi.org 10.5281/zenodo.5013542.

Members of the Darwin Tree of Life Consortium are listed here: https://doi.org/10.5281/zenodo.4783559.

\section{Acknowledgements}

JWS thanks Caroline Ware, Nicky Reilly, Naomi Lake, and the NHM Wildlife Garden Team for permitting specimen collection. JWS also thanks Donney Nicholson for assistance with tissue shipment as well as David Gower, Simon Loader (Department of Life Sciences), and John Tweddle (Angela Marmont Centre) for logistical assistance.
Birstein V], Mazin AL: Chromosomal Polymorphism of Bufo Bufo: Karyotype and C-Banding Pattern of B. B. Verrucosissima. Genetica. 1982 Publisher Full Text

Carrier JA, Beebee TJC: Recent, Substantial, and Unexplained Declines of the Common Toad Bufo Bufo in Lowland England. Biological Conservation. 2003; 111(3): 395-99.

Publisher Full Text

Challis R, Richards E, Rajan J, et al.: BlobToolKit - Interactive Quality Assessment of Genome Assemblies. G3 (Bethesda). 2020; 10(4): 1361-74. PubMed Abstract | Publisher Full Text | Free Full Text

Chin CS, Peluso P, Sedlazeck FJ, et al.: Phased Diploid Genome Assembly with Single-Molecule Real-Time Sequencing. Nat Methods. 2016; 13(12): 1050-54. PubMed Abstract | Publisher Full Text | Free Full Text

Chow W, Brugger K, Caccamo M, et al:: gEVAL - a Web-Based Browser for Evaluating Genome Assemblies. Bioinformatics. 2016; 32(16): 2508-10. PubMed Abstract | Publisher Full Text | Free Full Text Dudchenko O, Batra SS, Omer AD, et al.: De Novo Assembly of the Aedes Aegypti Genome Using Hi-C Yields Chromosome-Length Scaffolds. Science. 2017: 356(6333): 92-95.

PubMed Abstract | Publisher Full Text | Free Full Text

Edwards RJ, Tuipulotu DE, Amos TG, et al.: Draft Genome Assembly of the Invasive Cane Toad, Rhinella Marina. Gigascience. 2018; 7(9): giy095.

PubMed Abstract | Publisher Full Text | Free Full Text

Ewert JP: The Neural Basis of Visually Guided Behavior. Sci Am. 1974; 230(3): 34-42.

PubMed Abstract | Publisher Full Text

Formenti G, Rhie A, Balacco J, et al:: Complete Vertebrate Mitogenomes Reveal Widespread Repeats and Gene Duplications. Genome Biol. 2021; 22(1): 120.

PubMed Abstract | Publisher Full Text | Free Full Text

Garrison E, Marth G: Haplotype-Based Variant Detection from Short-Read

Sequencing. 2012; arXiv: 1207.3907.

Reference Source

Gregory: Animal Genome Size Database. 2021.

Reference Source

Guan D, McCarthy SA, Wood J, et al.: Identifying and Removing Haplotypic Duplication in Primary Genome Assemblies. Bioinformatics. 2020; 36(9): 2896-98.

PubMed Abstract | Publisher Full Text | Free Full Text
Howe K, Chow W, Collins J, et al.: Significantly Improving the Quality of Genome Assemblies through Curation. Gigascience. 2021; 10(1): giaa153. PubMed Abstract | Publisher Full Text | Free Full Text Kerpedjiev P, Abdennur N, Lekschas F, et al:: HiGlass: Web-Based Visual Exploration and Analysis of Genome Interaction Maps. Genome Biol. 2018. 19(1): 125.

PubMed Abstract | Publisher Full Text | Free Full Text

Lu B, Jiang J, Wu H, et al.: A Large Genome with Chromosome-Scale Assembly Sheds Light on the Evolutionary Success of a True Toad (Bufo Gargarizans). Mol Ecol Resour. 2021; 21(4): 1256-73.

PubMed Abstract | Publisher Full Text

Makino S, and Others: An Atlas of the Chromosome Numbers in Animals. An Atlas of the Chromosome Numbers in Animals., no. 2nd ed.(1st American ed.) 1951.

Reference Source

Petrovan SO, Schmidt BR: Volunteer Conservation Action Data Reveals Large-Scale and Long-Term Negative Population Trends of a Widespread Amphibian, the Common Toad (Bufo Bufo). PLoS One. 2016; 11(10): e0161943. PubMed Abstract | Publisher Full Text | Free Full Text

Pruitt KD, Brown GR, Hiatt SM, et al:: RefSeq: An Update on Mammalian Reference Sequences. Nucleic Acids Res. 2014; 42(Database issue): D756-63. PubMed Abstract | Publisher Full Text | Free Full Text

Rao SSP, Huntley MH, Durand NC, et al.: A 3D Map of the Human Genome at Kilobase Resolution Reveals Principles of Chromatin Looping. Cell. 2014; 159(7): 1665-80.

PubMed Abstract | Publisher Full Text | Free Full Text

Rhie A, McCarthy SA, Fedrigo O, et al:: Towards Complete and Error-Free Genome Assemblies of All Vertebrate Species. Nature. 2021; 592(7856): 737-46.

PubMed Abstract | Publisher Full Text | Free Full Text

Shaheen J, Mudd AB, Diekwisch TGH, et al.: Pseudogenized Amelogenin Reveals Early Tooth Loss in True Toads (Anura: Bufonidae). Integr Comp Biol. 2021; icab039.

PubMed Abstract | Publisher Full Text

Simão FA, Waterhouse RM, Ioannidis P, et al: : BUSCO: Assessing Genome Assembly and Annotation Completeness with Single-Copy Orthologs. Bioinformatics. 2015; 31(19): 3210-12.

PubMed Abstract | Publisher Full Tex 


\section{Open Peer Review}

\section{Current Peer Review Status:}

\section{Version 1}

Reviewer Report 04 January 2022

https://doi.org/10.21956/wellcomeopenres.19125.r46560

(c) 2022 Shaffer $\mathbf{H}$. This is an open access peer review report distributed under the terms of the Creative Commons Attribution License, which permits unrestricted use, distribution, and reproduction in any medium, provided the original work is properly cited.

\section{H. Bradley Shaffer}

Department of Ecology and Evolutionary Biology, UCLA La Kretz Center for California Conservation Science, Institute of the Environment and Sustainability, University of California, Los Angeles, Los Angeles, CA, USA

This is a straightforward genome release report of an important European anuran. The methods and presentation of the data are obviously formulaic, but in this case, that's fine. It could be indexed as is, although I have a few small comments that might increase its visibility or clarity, particularly for the amphibian community that will be its primary user group.

The Introduction is very thin and not really very informative or compelling. I would include more on the family Bufonidae, for example. It is the third most speciose family out of 54 currently recognized on Amphibiaweb, there's been a lot of recent controversy over genus-level taxonomy that kind of thing. I'd like to see a bit more on conservation, given how hard toads have been hit by chytrid. What about the portion of the family phylogeny that is now covered by the three species with genomes, and important targets for additional lineages?

\section{Methods:}

Usually one records snout-urostyle length in an anuran, rather than snout-vent. Or at least that's the measurement I prefer. They are almost identical.

I could live without Figure 4, but it's fine if that is what you want. I don't think it's terribly necessary.

\section{Small notes:}

Benthic larvae - I've never heard that term for anuran larva. Unless they are very specialized, it applies to pretty much all tadpoles. I would drop this.

Last sentence of the Genome Annotation section should be corrected to say “...as described by Pruitt et al. (2014):

"The annotation (NCBI Bufo bufo Annotation Release 100; Table 1) was generated from 
transcripts and proteins retrieved from NCBI Entrez by alignment to the genome assembly, as described by (Pruitt et al., 2014)."

Is the rationale for creating the dataset(s) clearly described?

Partly

Are the protocols appropriate and is the work technically sound?

Yes

Are sufficient details of methods and materials provided to allow replication by others? Yes

Are the datasets clearly presented in a useable and accessible format?

Yes

Competing Interests: No competing interests were disclosed.

Reviewer Expertise: Amphibian and reptile evolution and ecology; conservation genomics and genetics; conservation biology

I confirm that I have read this submission and believe that I have an appropriate level of expertise to confirm that it is of an acceptable scientific standard.

Reviewer Report 01 November 2021

https://doi.org/10.21956/wellcomeopenres.19125.r46626

(c) 2021 Cai Z. This is an open access peer review report distributed under the terms of the Creative Commons Attribution License, which permits unrestricted use, distribution, and reproduction in any medium, provided the original work is properly cited.

\section{Zexi Cai}

Center for Quantitative Genetics and Genomics, Aarhus University, Tjele, Denmark

The authors presented a high quality genome assembly of the common toad. The data generation and analysis pipelines applied in this report are sound and the information for the resource reuse are clearly stated in the report.

However, in the "Genome annotation" part, the authors stated the method which should go to method part instead of a summary of the annotated genomic feature. Also, in the method part, the detail of running BUSCO should be stated clearly.

Is the rationale for creating the dataset(s) clearly described?

Yes

Are the protocols appropriate and is the work technically sound? 
Yes

Are sufficient details of methods and materials provided to allow replication by others? Yes

Are the datasets clearly presented in a useable and accessible format?

Yes

Competing Interests: No competing interests were disclosed.

Reviewer Expertise: Bioinformatics, quantitative genetics and genomics.

I confirm that I have read this submission and believe that I have an appropriate level of expertise to confirm that it is of an acceptable scientific standard. 